\title{
Large fibroadenoma mimicking malignancy
}

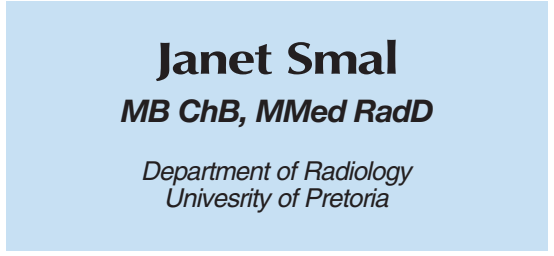

\section{Introduction}

Very large fibroadenomas can occur in young patients with ominous looking features that can mimic malignancy.

\section{Case report}

A 25-year-old woman complained about a large painful mass in her right breast. Due to the fact that there was a positive family history of breast carcinoma and the mass was quite large, irregular and very firm on palpation, the patient was sent for mammography and ultrasound examination.

On mammography a large, irregular and dense mass was noted in the superior lateral quadrant of the right breast. No calcifications were detected.

On ultrasound examination an irregular, lobulated, large mass was detected in the superior lateral quadrant of the right breast. The mass had an inhomogeneous texture, more hyperechoic, with irregular posterior acoustic enhancement and shadowing, especially at the edges of the mass.

The mass measured $>5 \mathrm{~cm}$ in length and $>3 \mathrm{~cm}$ in depth. No characteristic calcifications were detected (Figs. 1a and b).

Due to the size, irregularity and

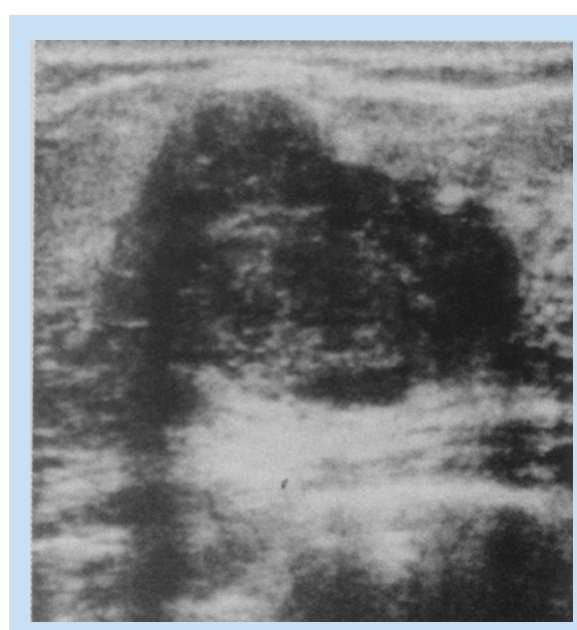

Fig. 1a. Transverse section of fibroadenoma on ultrasound.

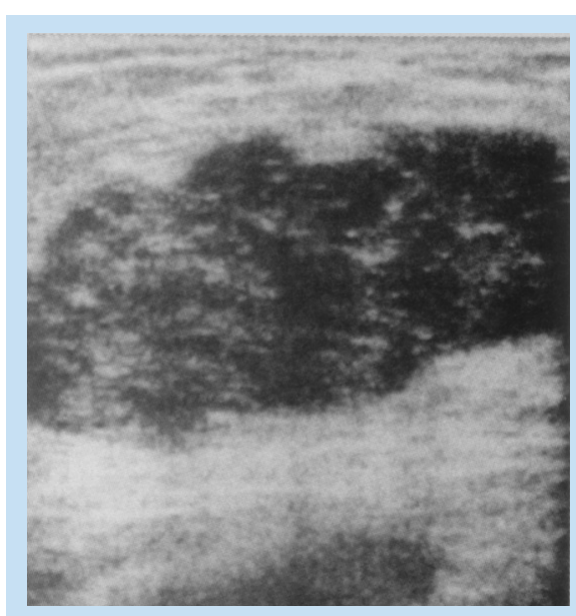

Fig. 1b. Longitudinal section of fibroadenoma on ultrasound.

density of the mass and the positive family history, the fear of malignancy was justified.

An excision biopsy was performed. Histological diagnosis confirmed a fibroadenoma.

\section{Discussion}

Fibroadenoma is an oestrogen- induced benign tumour, which develops during adolescence. Pregnancy and lactation are growth stimulants, with regression after menopause.

Patients have a mean age of 39 years (range $13-80$ years). These tumours may occur in postmenopausal women receiving oestrogen replacement therapy.

Clinically it is a firm, smooth, sometimes lobulated freely moveable mass, rarely tender or painful.

Sizes vary between $1 \mathrm{~cm}$ and $5 \mathrm{~cm}$ in diameter (in $60 \%$ of cases), tumours are multiple in $10-20 \%$, and bilateral in $4 \%$ of cases.

\section{Ultrasound characteristics}

Ultrasound characteristics are as follows: (i) round or oval mass with length-to-depth ratio $>1.4$ (carcinomas usually $<1.4$ ); (ii) hypoechoic or hyperechoic or mixed pattern or anechoic or isoechoic compared with adjacent fibroglandular tissue; (iii) homogeneous or inhomogeneous texture with regular, lobulated or irregular contour; (iv) there can be posterior acoustic enhancement or acoustic shadowing without calcification; and $(v)$ an echogenic halo with lateral shadowing can also be present.

A giant fibroadenoma $(>6 \mathrm{~cm})$ can occur in adolescents or young adults. ${ }^{2}$

\section{Differential diagnosis}

Medullary, mucinous or papillary carcinoma or carcinoma within the fibroadenoma.

\section{References}

1. Sutton D, Young JWR, A Short Textbook of Clinical Imaging. London: Springer Verlag, 1990: 415-424.

2. Dahnert W. Radiology Review Manual. 2nd ed. Baltimore: Williams and Wilkins, 1993: 349. 